\title{
Authoring for Interactive Storytelling Workshop
}

\author{
Charlie Hargood ${ }^{1}$, Alex Mitchell ${ }^{2}$, David E. Millard ${ }^{3}$, and Ulrike Spierling ${ }^{4}$ \\ 1 Bournemouth University \\ chargood@bournemouth.ac.uk \\ 2 National University of Singapore \\ alexm@nus.edu.sg \\ 3 University of Southampton \\ dem@ecs.soton.ac.uk \\ 4 RheinMain University of Applied Sciences \\ ulrike.spierling@hs-rm.de
}

\begin{abstract}
One of the most significant challenges facing narrative systems research is the authoring of interactive storytelling, and the processes and technology to support it. In this workshop we propose to host a discussion and presented new work in this space from researchers in creative and technical domains from both the Hypertext and Interactive Storytelling communities.
\end{abstract}

\section{Topic, Goals, and Expected Outcomes}

The authoring of interactive storytelling, and the processes and technology to support it, remains one of the most significant challenges facing narrative systems research. This workshop aims to bring together creatives, technologists, and associated researchers in a collective meeting to share research and advances in this space. Relevant work includes authoring tools, methodologies for authorship, frameworks and technology for assisting writers, and experimental paradigms of interactive storytelling creation such as locative storytelling and AR narrative.

The workshop aims to:

- Create a meeting venue for active researchers in this area to come together and share their work

- Foster a community around this work, as a step towards future collaboration

- Identify substantial challenges in this area that may be collectively targeted going forward

- Provide a venue for publication of early work in this space

As well as the above aims, this workshop also aims to bridge the gap within the narrative systems research community between the interactive storytelling community (whose natural venue is ICIDS) and the Hypertext Narrative community (whose natural venue is ACM Hypertext). Both communities have been exploring interactive storytelling authorship in recent years, and it is our hope that a workshop organised by researchers from both communities can provide a focal point for dialogue and collaboration. Two of the organisers have run 
several workshops, both separately (Spierling at ICIDS 2008, 2009 and 2010) and together (Mitchell and Spierling at ICIDS 2014 and 2016) on similar topics. Similarly, the other two organizers (Hargood and Millard) have run a successful narrative systems workshop series at ACM Hypertext since $2011^{5}$. The proposed workshop aims to form the basis of a workshop series, following on from the earlier ICIDS workshops, acting as a sister workshop series to the NHT workshop series and potentially forming a connection between the two communities. This would be a notable outcome, and may in future lead to a special issue journal publication fed by contributions from both workshops.

\section{Format and Proposed Schedule}

Our proposal is for a Half Day workshop.

We propose a mixture of a structured paper session and a less structured "unconference" session. Paper sessions provide an opportunity for young researchers to publish their early work and for early position papers on new ideas from more established researchers for work that is not yet ready or mature enough for the main conference. The purpose of these works is to gather community feedback on early progress, and stimulate discussion with new ideas in the area. An "unconference" can be defined as: "a meeting for which the agenda is defined by the attendees at the start of the meeting". In this case a series of proposed discussions, activities, and meetings will be gathered over the day and this will be formed into plenary discussions and/or spin out meetings in the unconference session. This gives attendees an opportunity to have more specific meetings on targeted areas and to use the workshop as a platform to host collaborations and conversations inspired by the earlier part of the workshop. The organisers have been using this format of formal presentation followed by unconference in a similar workshop series (NHT) with repeated success.

A draft schedule for the workshop is as follows:

\begin{tabular}{|c|c|}
\hline Time & Session \\
\hline $12.00-13.00$ & Lunch \\
\hline $13.00-13.15$ & Welcome \\
\hline $13.15-14.30$ & Presentation Session \\
\hline $14.30-15.00$ & Coffee \\
\hline $15.00-17.00$ & Unconference \\
\hline $17.00-17.15$ & Conclusions and Close \\
\hline
\end{tabular}

Other than seating, tables, projector, and white board, no special equipment or resources are needed for this workshop.

\footnotetext{
${ }^{5}$ http://nht.ecs.soton.ac.uk
} 


\section{Call for Participation (Draft)}

Authoring for Interactive Storytelling 2017 (AIS'17)

A Workshop@ICIDS'17 in Madeira, 14th Nov2017

This call for participation is seeking submitted papers and attendance at the Authoring for Interactive Storytelling workshop held at ICIDS 2017 in Madeira. This workshop seeks to provide a venue for researchers in the area of interactive digital narrative authoring and narrative systems to share early work, new ideas, and identify challenges facing the field, with a view to fostering collaboration and the formation of a coherent research community in this space.

This workshop will provide structured paper presentations and unstructured "unconference" sessions, the content of which will be dictated by the workshop attendees during the workshop session itself. Papers submitted to this workshop should be 3-6 pages long, and in Springer LNCS format. Papers will be peer reviewed, and successful submissions will presented at the workshop. There is also the potential that papers accepted to the workshop will be published as part of the workshop proceedings. Submissions should be sent to chargood@bournemouth.ac.uk by midnight on 15th September. Topics relevant to this workshop include:

- Authoring tools

- Frameworks for interactive digital narrative authoring

- Digital writing methodologies

- Co-Design of interactive digital narrative

- Locative and in-situ authoring

- Experimental digital writing paradigms

- New media creation and production

- Assisting technology for writers

Key Dates:

- Submissions due: 15th September

- Notification due: 29th September

- Workshop: 14th November 2017

Organisers:

- Charlie Hargood - chargood@bournemouth.ac.uk

- Alex Mitchell - alexm@nus.edu.sg

- David Millard - dem@ecs.soton.ac.uk

- Ulrike Spierling - ulrike.spierling@hs-rm.de

\section{Participants}

Our participants will most likely come from the following groups: 
- PhD Students and Early Career Researchers presenting their work on interactive digital narrative authoring

- Academics, Researchers, Writers, and members of the creative industries interested in interactive digital narrative authoring

We expect a maximum of 40 attendees, with a likely attendance of approximately 15-25. This is based on the organisers' experience of running similar workshops such as NHT and the earlier ICIDS workshops.

\section{Organising Committee}

\subsection{Organisers}

This workshop's organisers are academics from the wider interactive storytelling and narrative systems community. All have experience in running academic workshops (Hargood and Millard have run NHT since 2011, and Mitchell and Spierling have run previous versions of ICIDS authoring workshops in 2008, 2009, 2010, 2014 and 2016).

- Charlie Hargood - Bournemouth University - chargood@bournemouth.ac.uk

- Alex Mitchell - National University of Singapore - alexm@nus.edu.sg

- David Millard - University of Southampton - dem@ecs.soton.ac.uk

- Ulrike Spierling - Hochschule RheinMain, University of Applied Sciences ulrike.spierling@hs-rm.de

\subsection{Potential Programme Committee}

The following represents a likely programme committee in addition to the organisers (though not yet confirmed/consulted).

- Mark Bernstein - Eastgate Systems

- Fred Charles - Bournemouth University

- Rosamund Davies - Greenwich University

- Mads Haahr - Trinity College Dublin

- Ido Iurgel - Rhine-Waal University of Applied Sciences

- Stacey Mason - UCSC

- Valentina Nisi - Madeira Interactive Technologies Institute

- Nicolas Szilas - TECFA, University of Geneva

- Mark Weal - University of Southampton 